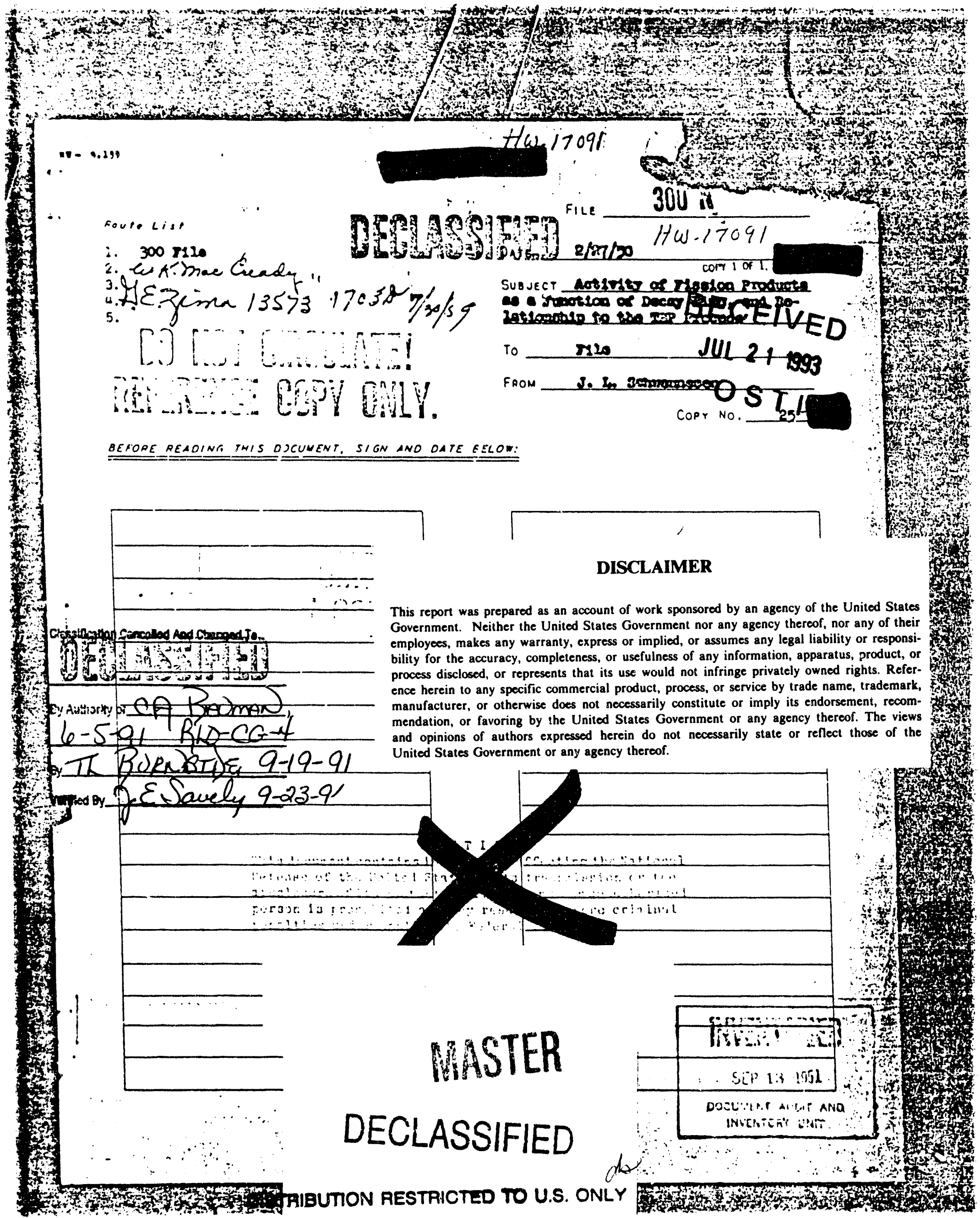




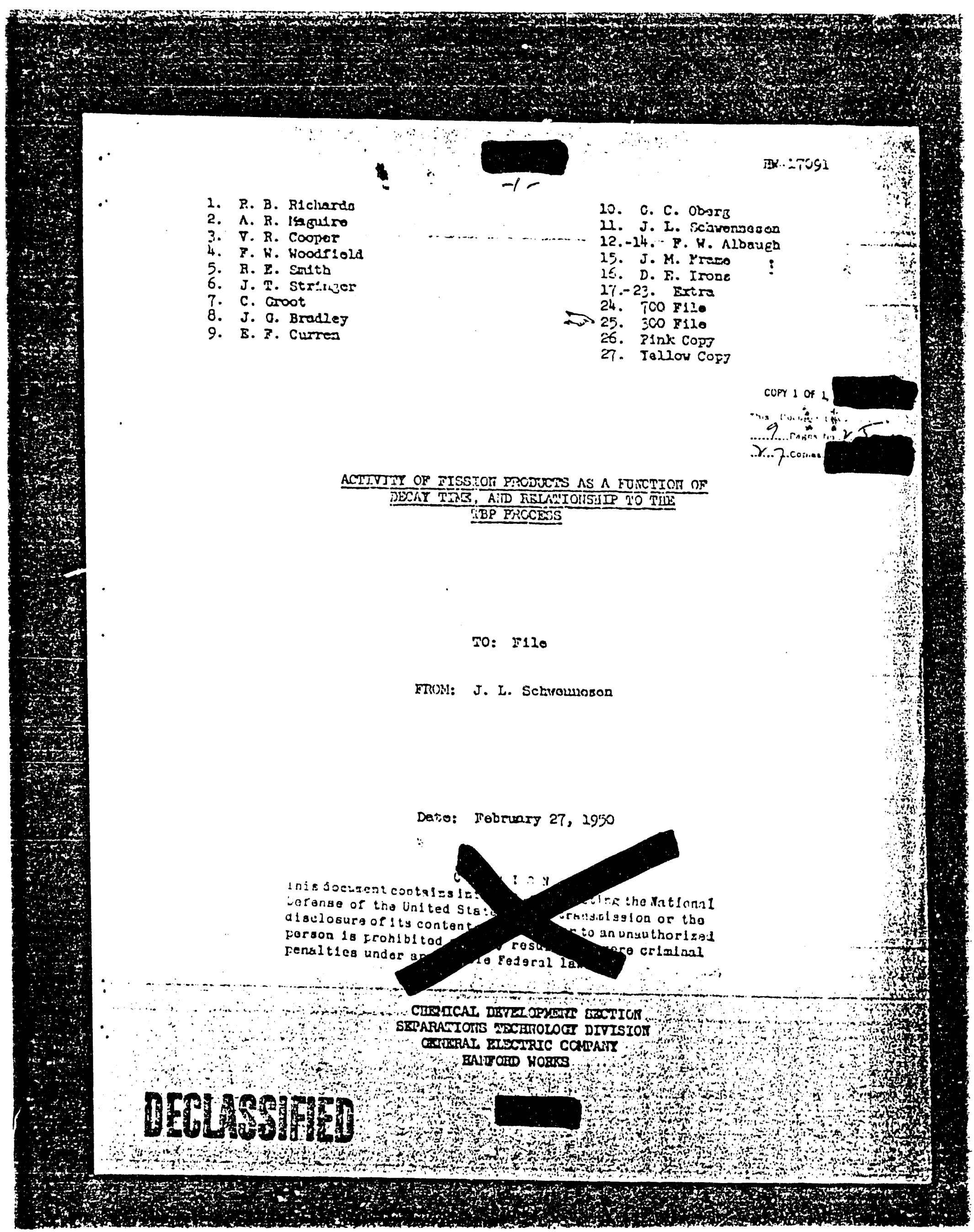




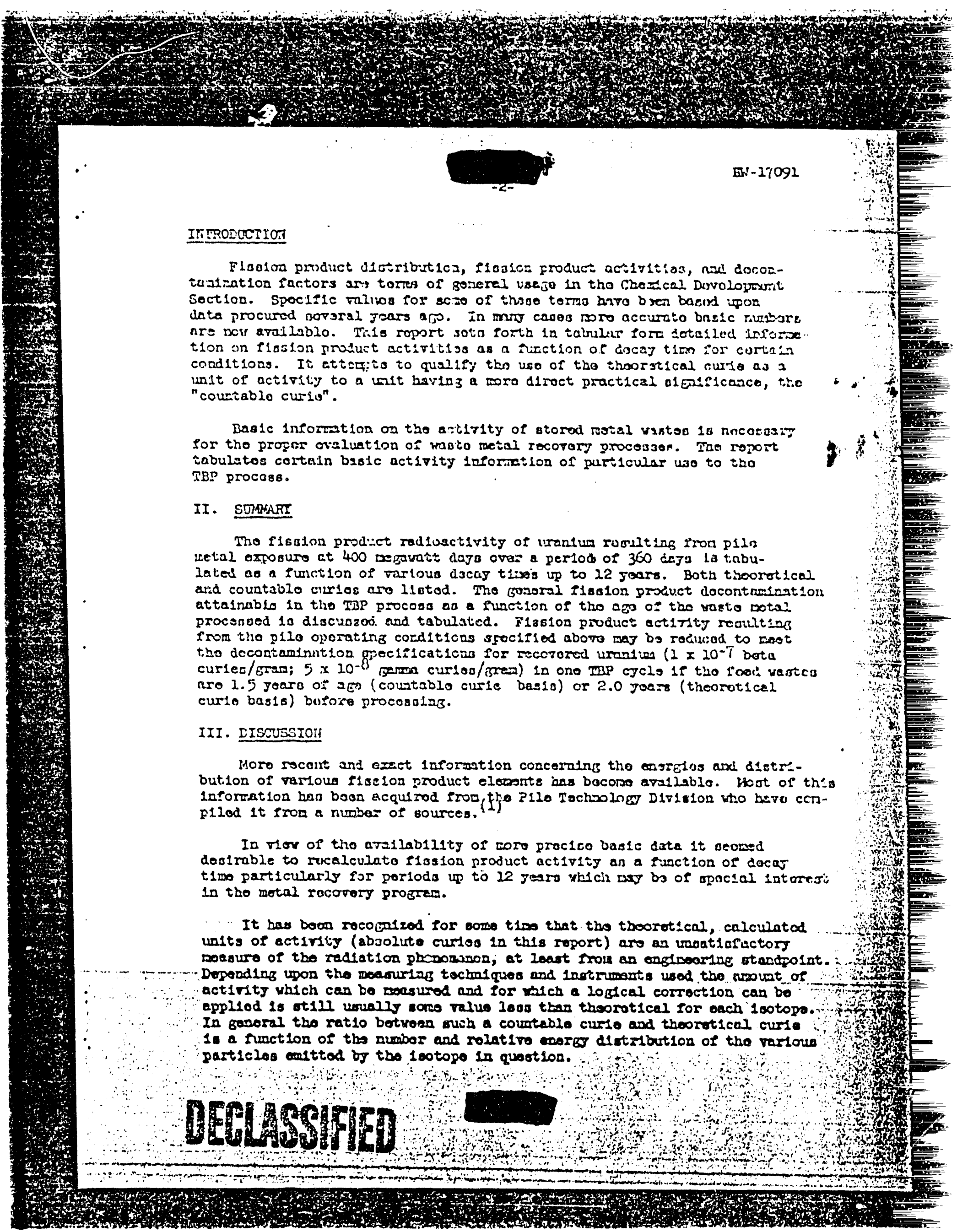




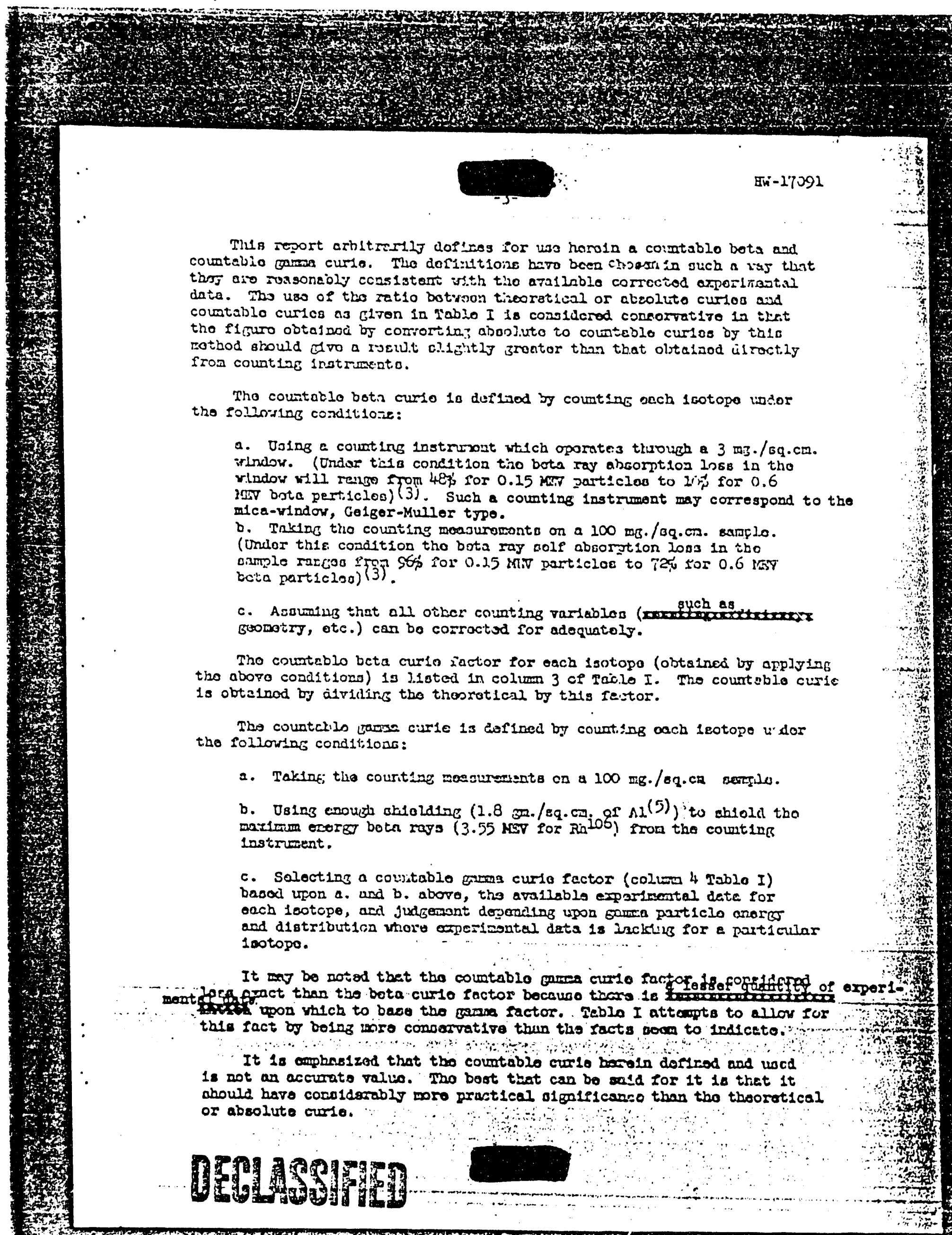




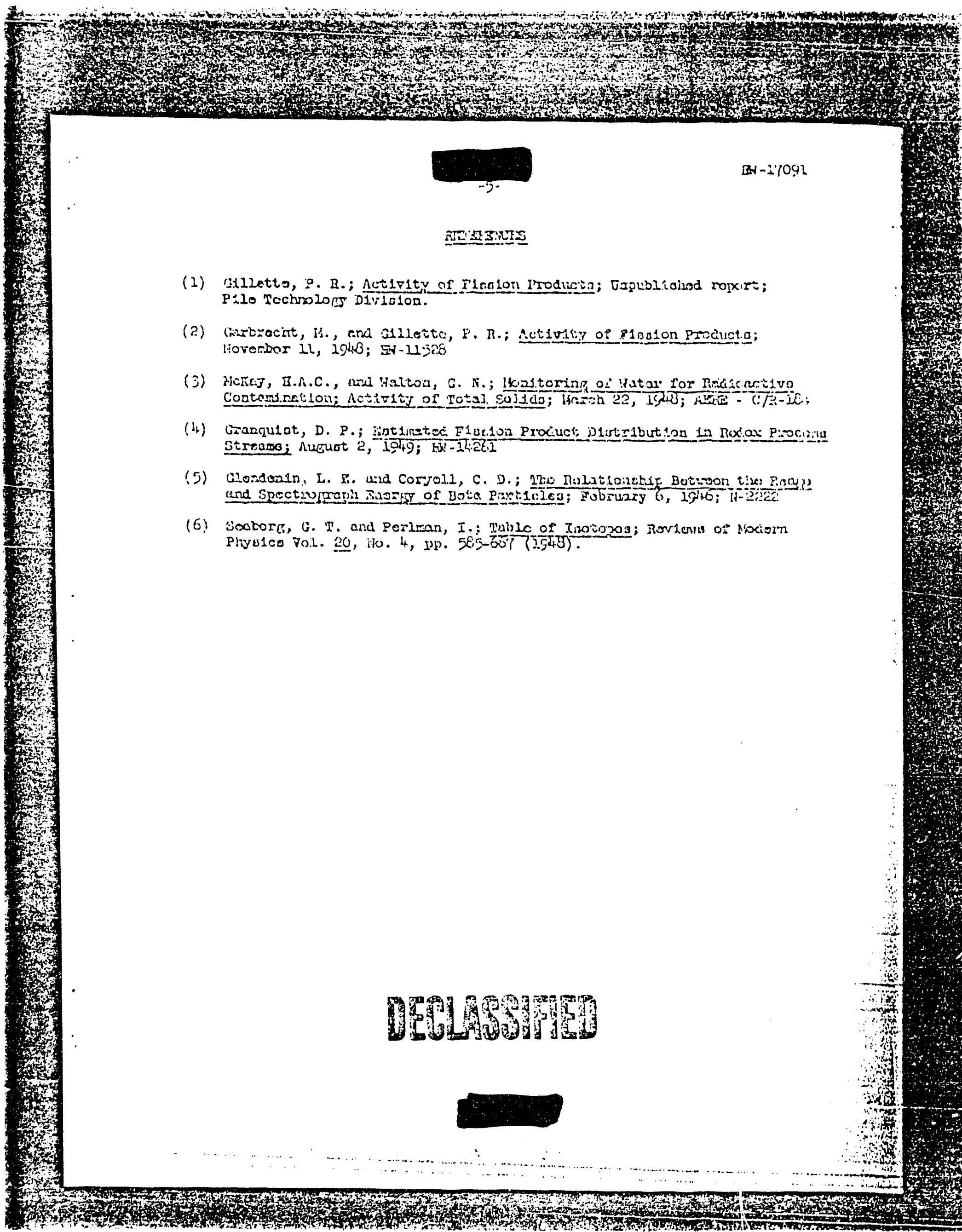




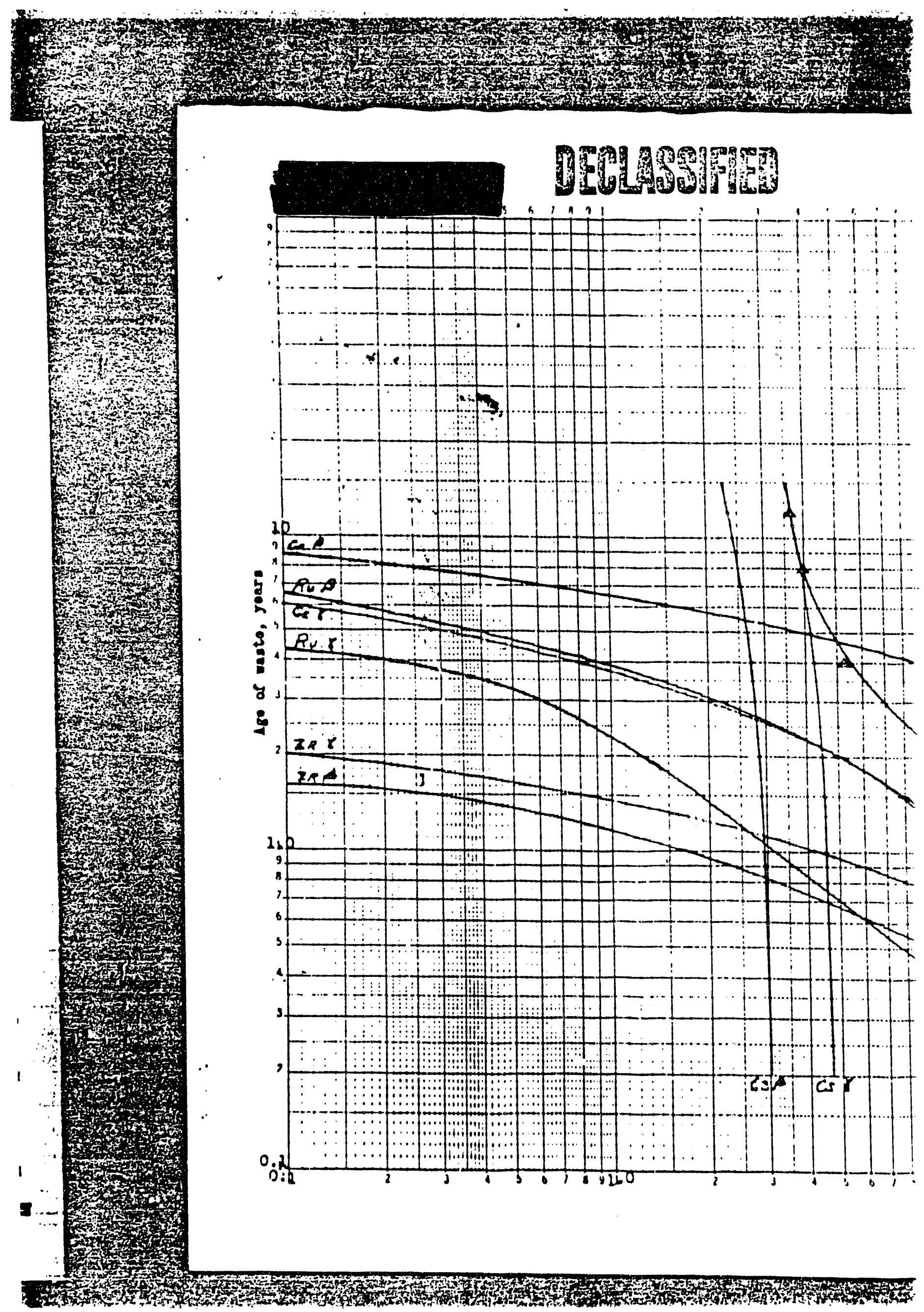



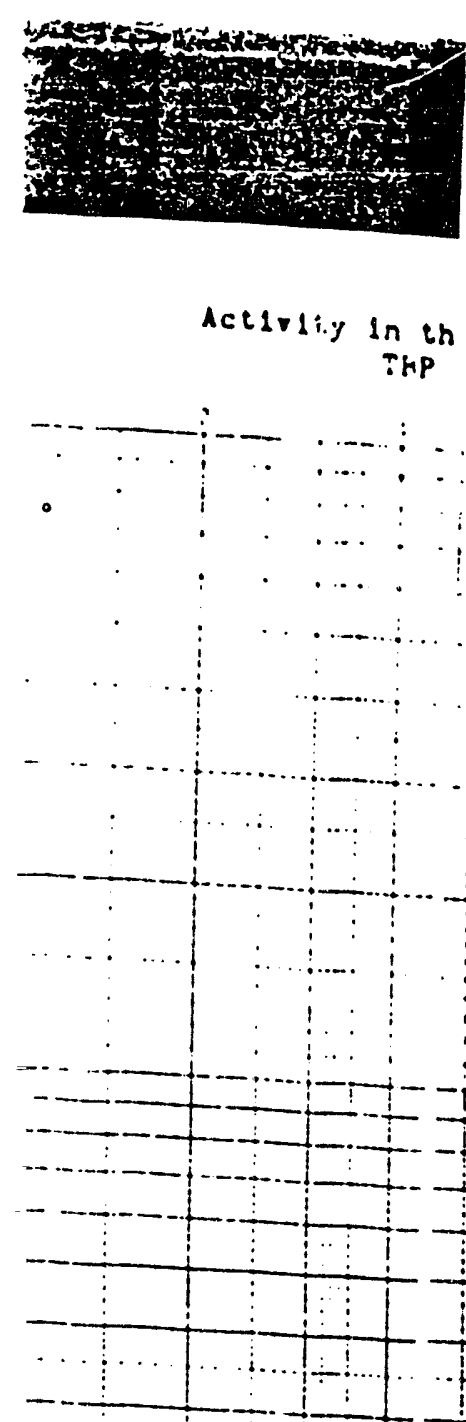

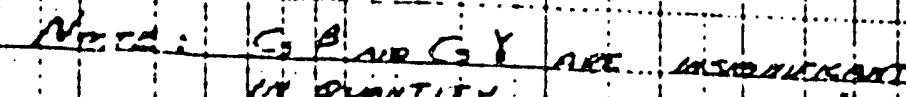

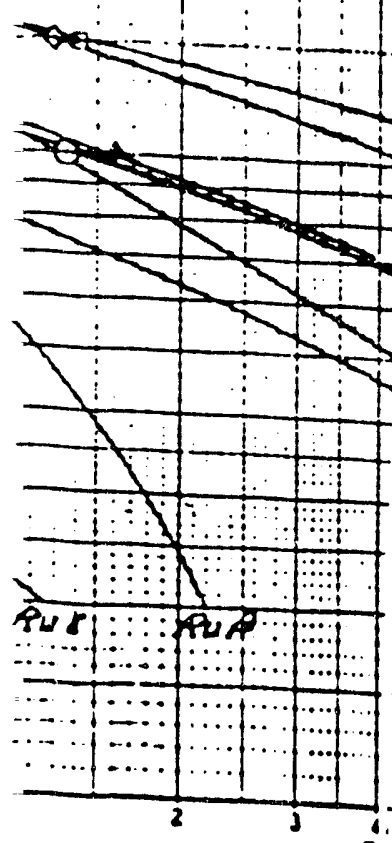

\section{ILoure 2}

\section{t}




\begin{tabular}{|c|c|c|c|c|c|c|c|c|c|c|c|c|}
\hline \multicolumn{4}{|c|}{$1 \mathrm{Ir.}$} & \multicolumn{4}{|c|}{$2 \mathrm{Ir}}$. & \multicolumn{4}{|c|}{$4 I r}$. & \multirow{3}{*}{ do } \\
\hline \multicolumn{4}{|c|}{ Aba furter conat Curtee } & \multicolumn{2}{|c|}{ Abo. Cur1es } & \multicolumn{2}{|c|}{ Coont: Curlno } & \multicolumn{4}{|c|}{ Absearles founie furdes } & \\
\hline 3 & $r$ & $\boldsymbol{B}$ & $\gamma$ & 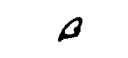 & $\gamma$ & $a^{\prime}$ & 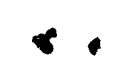 & $\boldsymbol{\beta}$ & $\gamma$ & $\beta$ & $r$ & \\
\hline 6.23 & - & 3.18 & - & 0.05 & - & 0.04 & - & & & - & & - \\
\hline 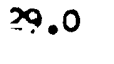 & - & 16.47 & - & 28.2 & - & 16.02 & - & 26.7 & - & 15.27 & - & 2 \\
\hline 6.1 & - & 4.69 & - & 0.08 & - & 0.06 & - & - & - & - & 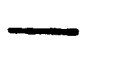 & - \\
\hline 12.4 & 21.0 & 1.68 & 4.36 & .265 & 0.50 & 0.04 & 0.10 & & & - & - & $=$ \\
\hline 30.1 & 30.5 & .93 & 12.2 & .64 & 0.65 & 0.02 & 0.26 & & - & 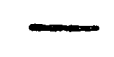 & - & - \\
\hline 1.01 & .50 & .20 & .17 & - & - & - & - & - & - & - & - & - \\
\hline 12.6 & 4.29 & 12.06 & 2.84 & 5.33 & 1.83 & 3.17 & 1.22 & .92 & .378 & .89 & .21 & . \\
\hline .19 & - & .21 & - & .03 & - &.$\infty 2$ & - & $1=$ & - & - & $\longrightarrow$ & $=$ \\
\hline .56 & 0.56 & .06 & .03 & 0.62 & 0.42 & .05 & .06 & .25 & .25 & .03 & .03 & - \\
\hline. $\mathbf{a r}$ & 0.06 . & . & . 28 & - & - & - & - & - & - & - & - & . \\
\hline 13.2 & 12.3 & 2.93 & 4.55 & 12.9 & 12.0 & 2.86 & 4.46 & 22.6 & $\mathcal{H} .5 \mathrm{k}$ & 2.76 & 4.27 & 11. \\
\hline- & & - & & & & - & & - & - & 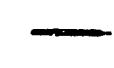 & - & . \\
\hline .06 & .06 & .01 &.$\infty$ & - & - & - & - & - & - & 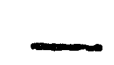 & & - \\
\hline . & 125. & 262. & 22.5 & 103 of & 69.7 & 56.5 & 4.97 & 16.46 & 7.92 & 8.90 & 0.79 & c \\
\hline 14.2 & & 2.64 & & 37.1 & & 2.22 & & 25.6 & - & 2.53 & - & $12 \mathrm{e}$ \\
\hline 1.77 & - & - & A & 1.77 & & - & $\ldots$ & 1.60 & $\longrightarrow$ & $\bar{T}$ & - & 1. \\
\hline 0.75 & 0.75 & - & 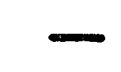 & 0.53 & 0.53 & $\longrightarrow$ & - & 0.27 & 0.27 & - & & 0. \\
\hline 35. & 196. & 107. & 36.8 & 191. & 65.6 & 83.0 & 21. & 8.2 & 20.3 & 29.3 & 5.30 & 69.6 \\
\hline
\end{tabular}




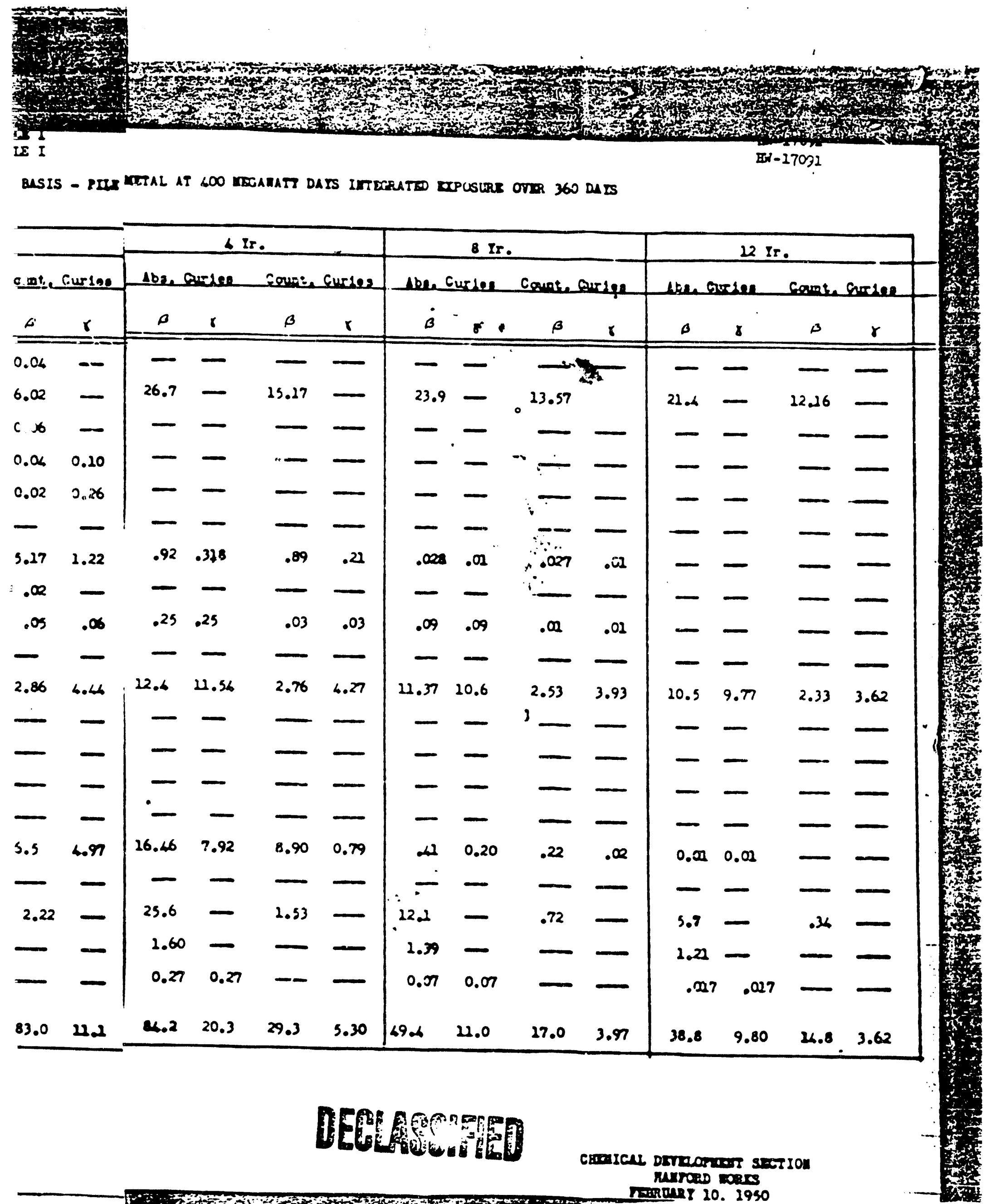


EXPECTID ACT ¿̈V

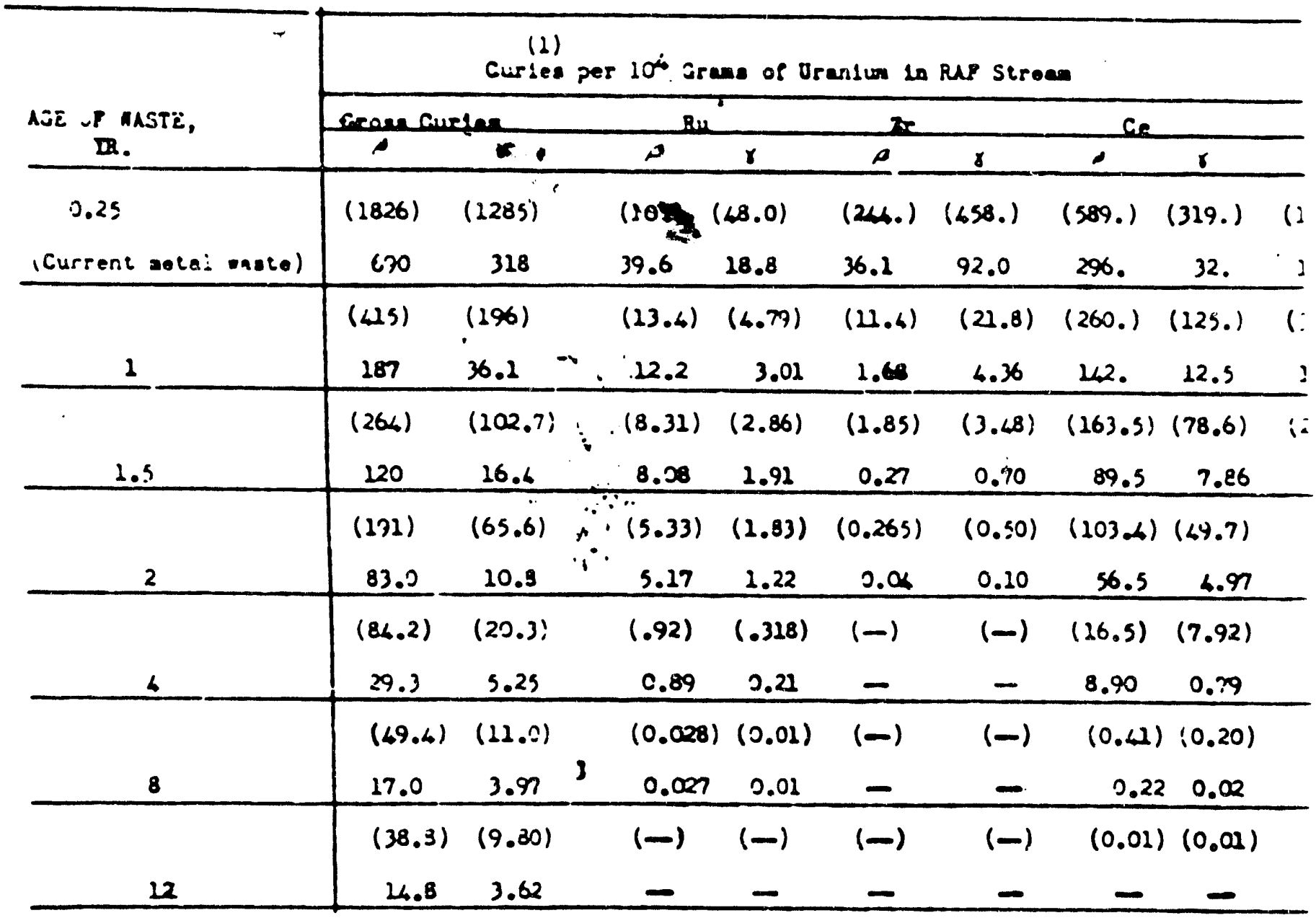

MOTES,

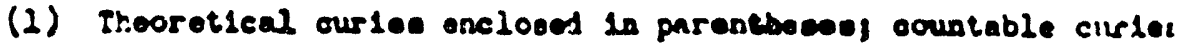

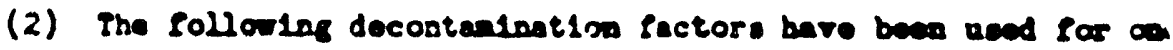

$$
\begin{aligned}
& -2 \times 20 \% \\
& 25-3 \times 10^{3} \\
& \begin{array}{l}
\text { Co, } 95,68-1 \times 106 \\
\text { ali othere }-1 \times 10^{6}
\end{array}
\end{aligned}
$$

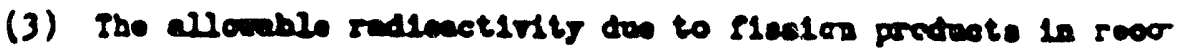

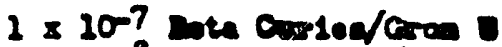

$$
\begin{aligned}
& 5 \times 10^{8} \text { onen ourlea/eren I }
\end{aligned}
$$

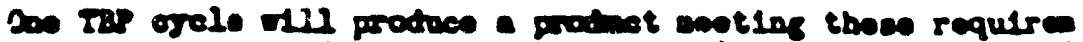
1. 1.5 jears old (countable pirs bele) or 2.0 joaro old ( 


\section{TAMSU}

itt cimiaution in tBP PRDCES

BASIS - PILE LETAL AT 600 XEEAAATT IAIS INTEEATDD EXPUSLHE OVER 360 DAYS

\begin{tabular}{l}
\hline \\
\hline
\end{tabular}

11arted Inedintely boantb.

I.: orelet

inced arange 108

ite is the lood mtarind

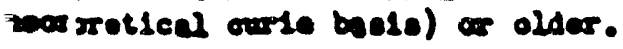


घ-17092

TT EAIT INTEEATDD EXPOSLRE OVER 360 DAYS

- por li fo Grans of Oraniza io RCO Strom

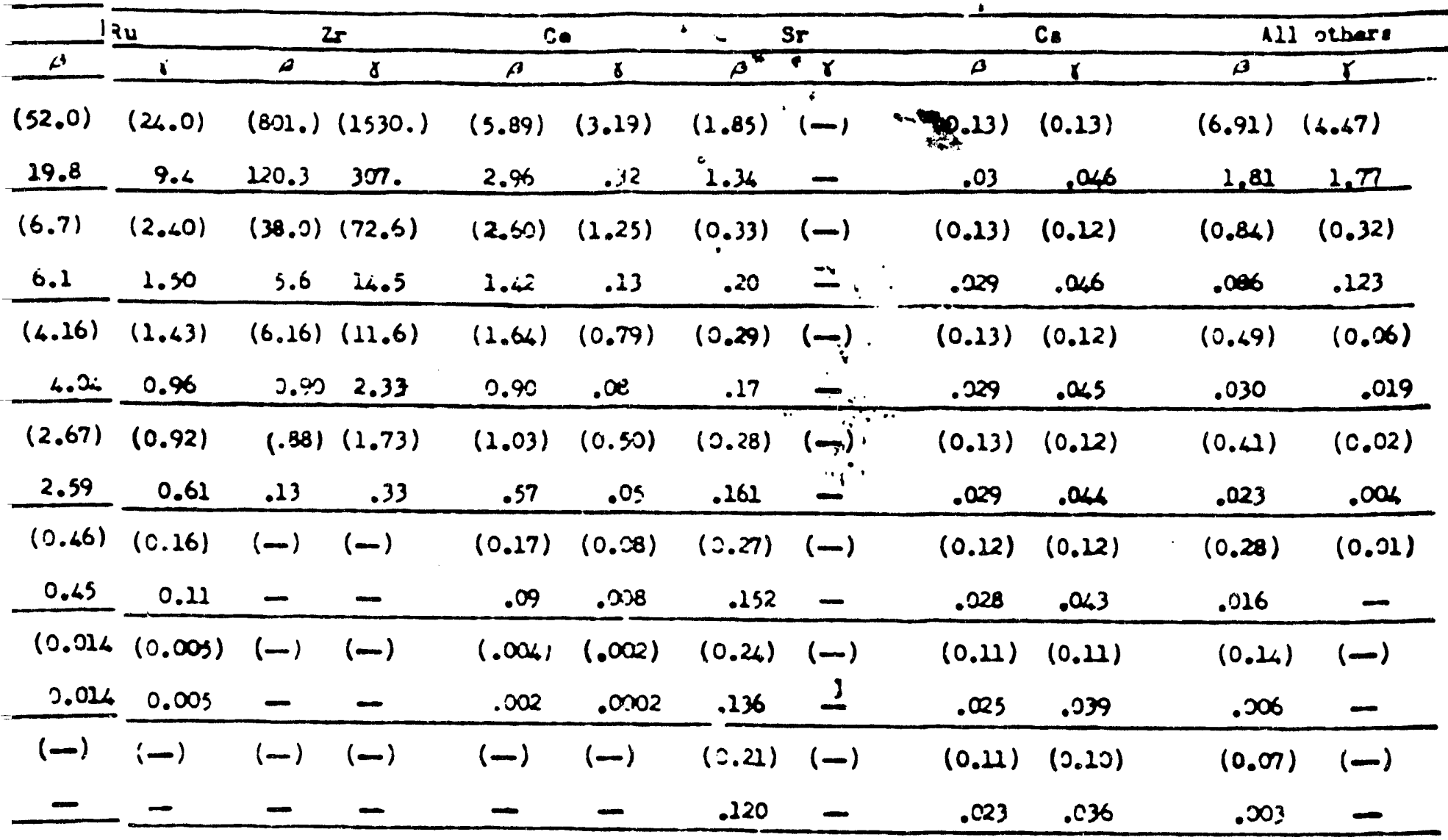

C:H-XICAI DEJELPUEAT SECTION HUNPOR MORXS

PRBHUAKI 10, 1950 

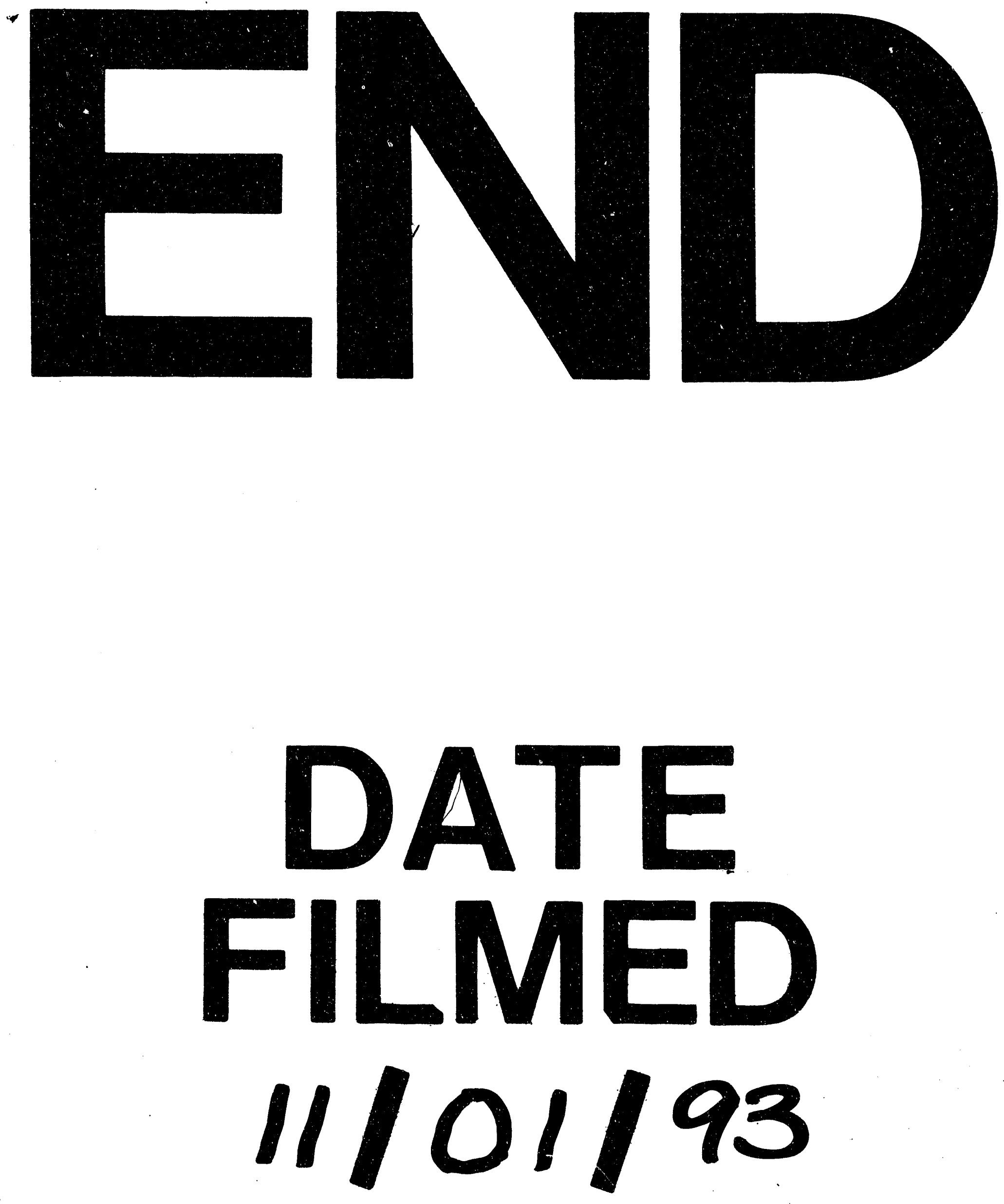


\section{,}

